Pacific Journal of Mathematics

DISTRIBUTION OF SQUAREFREE INTEGERS IN NON-LINEAR Ste 


\title{
DISTRIBUTION OF SQUAREFREE INTEGERS IN NON-LINEAR SEQUENCES
}

\author{
Ivan E. STUX
}

\begin{abstract}
I. This paper investigates the occurrences of the squarefree integers in sequences $s_{n}=[f(n)], n=1,2,3, \cdots$ where $f(x)$ belongs to classes of functions described by 'smoothness' conditions. The result obtained is an extention of the well known fact that $Q(x)=6 / \pi^{2} x+O\left(x^{1 / 2}\right)$, where $Q(x)=$ number of squarefree integers $\leqq x$; it states that $Q_{s}(x) \sim 6 / \pi^{2} g(x)$ where $Q_{s}(x)=$ number of squarefree integers $\leqq x$ in the sequence $s_{n}$, and $g(x)$ is the inverse function of $f(x)$.
\end{abstract}

This result relates to the deep theorem of Piateskii-Shapiro which states that if $1<c<12 / 11$ then the sequence $\left[n^{c}\right]$ has the proper rate of primes occuring, namely, $\pi_{c}(x) \sim x^{1 / 0} / \log x$.

The classes of functions used is described by the following:

DeFinItion 1. for given $1<c<2,0<\delta<1$

(1) $S(c, \delta)=$ set of functions $f(x)$ such that for some constant $a>0$ depending on $f$, and for sufficiently large $x$ 's, depending on $f$,

$$
\left(a x^{c}\right)^{(i)} \leqq(f(x))^{(i)}<\left(a x^{c+\delta}\right)^{(i)}
$$

holds for $i=0,1,2$, the superscripts indicating the $i^{\text {th }}$ derivative.

Functions like $x^{c}, 1<c<2$, or more generally $\sum_{i=1}^{k} a_{i} x^{c_{i}}(\log x)^{d_{i}}$, where the leading term has $a>0,1<c<2$, belong to these classes of functions.

The following theorem will be proved:

THEOREm 1. Let $1<c<4 / 3$, then there exists $a \delta_{c}=\delta(c)>0$, some small value depending on $c$ such that if $f(x) \in S(c, \delta), 0 \leqq \delta<$ $\delta(c)$, then

$$
Q_{s}(x)=6 / \pi^{-2} g(x)+O\left((g(x))^{1-s}\right)
$$

holds for some $\varepsilon>0$ depending on $c$ and $\delta$, where $Q_{s}(x)=$ number of $\left\{s_{n} \leqq x, s_{n}=[f(n)], s_{n}=\right.$ squarefree, $\left.n=1,2,3, \cdots\right\}, g(x)=$ inverse function of $f,[z]=$ integer part of $z$.

II. Following are the lemmas that will be used in the proof:

Lemma 1 (Piateskii-Shapiro, [2]). Let $f(x)$ be a continuously 
$g(x)$ be its inverse function. Then, for an integer $m$ such that $m=[f(n)]$, either $\{g(m)\}=0$ or $1-g^{\prime}(m-1)<\{g(m)\}<1$. Conversely, if $\{g(m)\}=0$ or $1-g^{\prime}(m+1)<\{g(m)\}<1$, then it follows that for some $n, m=[f(n)]$.

(The curly brackets indicate the fractional part of the real number, the straight brackets the integer part, as usual.)

Lemma 2 (a theorem of Erdös-Turán, [1]). If $\mu_{1}, \mu_{2}, \cdots$ is a real sequence and if $D_{N}$ denotes its discrepancy modulo one, then for each integer $m \geqq 1$ we have

$$
N D_{N^{*}} \leqq K\left(\frac{N}{m+1}+\sum_{t=1}^{m} \frac{1}{t}\left|\sum_{n=1}^{N} e\left(t \mu_{n}\right)\right|\right)
$$

(where $K$ is a constant and $e(z)=e^{2 \pi i z}$, as usual).

Lemma 3 (Van der Corput, pg, 64, [3]). Let $g(x)$ be a real function with a continuous and steadily decreasing derivative $g^{\prime}(x)$ in $(a, b)$, and let $g^{\prime}(b)=\alpha, g^{\prime}(a)=\beta$. Then

$$
\text { (4) } \sum_{a<n \leqq b} e(g(n))=\sum_{\alpha-r<<<\beta+\eta} \int_{a}^{b} e(g(x)-\nu x) d x+O(\log (\beta-\alpha+2))
$$

where $\eta$ is any positive constant less than one.

Lemma 4 (Van der Corput, pg. 61, [3]). Let $F(x)$ be a real function, twice differentiable, and let $F^{\prime \prime}(x) \geqq r>0$, or $F^{\prime \prime}(x) \leqq$ $-r<0$ throughout the interval $(a, b)$, then

$$
\left|\int_{a}^{b} e^{i F(x)} d x\right| \leqq \frac{8}{\sqrt{r}}
$$

III. The first part of the proof is aimed at establishing the uniform distribution modulo one and the discrepancy of that distribution for sequences $g(q)$ where $q$ are squarefree integers and $g(x)$ is the inverse function of a function in $S(c, \delta)$ (where $\delta$ is usually small, depending on $c$ ). The following is the result in this direction:

THEOREM 2. For given $1<c<2$, and $\delta>0$, small enough depending on $c$ alone, let $f(x) \in S(c, \delta)$ and let $g(x)$ be the inverse function of $f(x)$. Then the sequence $\{g(q): q \leqq K, q$ the squarefree integers\} is uniformly distributed modulo one and

$$
N(K, \xi)=\xi Q(K)+Q(K) D_{Q(K)}(g),
$$

and 
differentiable function with $f^{\prime}(x)>0, f^{\prime \prime}(x) \geqq 0$, for $x \geqq 1$, and let

$$
Q(K) D_{Q(K)}(g) \ll K^{3 / 5+(c+2 \delta) /(5 c(c+\delta))}+K^{1-1 /(2 c(c+o))}
$$

where $Q(K)=$ number of squarefree integers $\leqq K, N(K, \xi)=$ number of elements in the sequence $g(q), q \leqq K, q$ squarefree, which fall into a fixed interval of length $\xi(<1)$ modulo one, and $D_{Q(K)}(g)$ is the discrepancy, modulo one, of the sequence $g(q)$.

Clearly, uniform distribution holds whenever $\delta>0$ is small enough to make the exponents in the estimate (7) less than one.

Proof. For $h \geqq 1$, consider

$$
T_{h}(K)=\sum_{\substack{q \leq K \\ q \text { squarefree }}} e(h g(q)), \quad e(z)=e^{2 \pi i z} .
$$

Suppose that $K_{0}$ is the large value from where on the estimates of $g, g^{\prime}, g^{\prime \prime}$ induced by the definition 1 hold, and let $K>K_{0}$, then

$$
T_{h}(K)=\sum_{\substack{K_{0}<q \leq K \\ q \text { squarefree }}} e(h g(q))+O\left(K_{0}\right),
$$

and

$$
\sum_{\substack{K_{0}<q \leq K \\ q \text { squarefree }}} e(h g(q))=\sum_{\substack{K_{0}<n \leq K \\ n=\text { integer }}} e(h g(n)) \sum_{d^{2} \mid\left(n, P^{2}\right)} \mu(d)
$$

where $p=\Pi_{p \leqq K^{1 / 2}} p, p=$ primes, $(a, b)=$ greatest common divisor, $\mu(d)=$ Möbius function. We can further write

$$
\begin{aligned}
& \sum_{d \leqq \sqrt{K} \bar{K}} \mu(d) \sum_{K_{0} / d^{2}<m \leqq K / d^{2}} e\left(h g\left(d^{2} m\right)\right) \\
& =\sum_{\substack{d \leq A \\
d \backslash P}} \mu(d) \sum_{K_{0} ; d^{2}<m \leqq K \mid d^{2}} e\left(h g\left(d^{2} m\right)\right)
\end{aligned}
$$

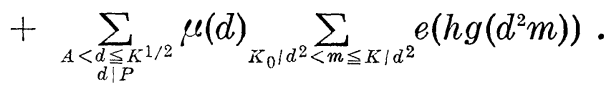

We will pick the value of $A$ later. The second sum in (10) can be estimated trivially as

$$
\sum_{A<d \leqq K^{1 / 2}}\left|\sum_{K_{0}^{\prime} d^{2}<m \leqq K^{\prime} d^{2}} e\left(h g\left(d^{2} m\right)\right)\right| \ll \sum_{A<d \leqq K^{1 / 2}} \frac{K}{d^{2}} \ll \frac{K}{A} .
$$

The first sum, on the other hand, is estimated by

$$
\sum_{d \leqq A}\left|\sum_{K_{0} / d^{2}<m \leqq K / d^{2}} e\left(h g\left(d^{2} m\right)\right)\right| .
$$

To estimate the inner sum, divide the interval $K_{0} / d^{2}<m<K / d^{2}$ up into pieces of type $1 / 2^{r} K / d^{2}<m \leqq 1 / 2^{r-1} K / d^{2}$, to get 


$$
\sum_{d \leqq A} \sum_{r}\left|\sum_{\substack{1 / 2^{r} K i d^{2}<m \leqq 1 / 2^{r-1} K / d^{2} \\ m \geqq N_{0} / d^{2}}} e\left(h g\left(d^{2} m\right)\right)\right| .
$$

We will estimate the last inner sum by using Lemma 3 and then Lemma 4. The conditions in definition 1 give that

$$
\left(\frac{y}{a}\right)^{(c+\delta)^{-1}}<g(y) \leqq\left(\frac{y}{a}\right)^{1 / c}, \frac{1}{c+\delta}\left(\frac{y}{a}\right)^{(c+\delta)^{-1}-1}<g^{\prime}(y) \leqq \frac{1}{c}\left(\frac{y}{a}\right)^{1 / c},
$$

the chain rule tells us that $(d / d x) g\left(d^{2} x\right)=[(d / d z) g(z)] \cdot d^{2}, z=d^{2} x$, and so we have, by Lemma 3, for each $r$

$$
\sum_{1 / 2^{r} K_{K / d^{2}<m \leqq 1 / 2^{r-1}}} e\left(h g\left(d^{2} m\right)\right)=\sum_{\nu} I_{\nu}+E,
$$

where the $\sum_{\nu}$ extends over $\left(1 / a_{1}(c+\delta)\right) h\left(\left(1 / 2^{r-1}\right) K\right)^{(c+\delta)^{-1-1}} d^{2}-1 / 2<$ $\nu<\left(1 / a_{2} c\right) h\left(\left(1 / 2^{r}\right) K\right)^{c-1-1} d^{2}+1 / 2, a_{1}=a^{(c+\delta)-1}, a_{2}=a^{1 / c}$, and

$$
I_{\nu}=\int_{2^{-r_{K}-2}}^{2^{-r+1} d^{-2}} e\left(h g\left(d^{2} x\right)-\nu x\right) d x,
$$

and

$$
E=O(\log (\max \nu-\min \nu+2)) .
$$

In (14), first we change variables to $y=d^{2} x$, and then apply Lemma 4

$$
I_{\nu}=\frac{1}{d^{2}} \int_{2^{-r} r_{K}}^{2^{-r+1} K} e\left(h g(y)-\frac{\nu y}{d^{2}}\right) d y
$$

but here $d^{2} / d y^{2}\left(h g(y)-\nu y / d^{2}\right) \geqq(c+\delta)^{-1}\left((c+\delta)^{-1}-1\right)(1 / a) h y^{(c+\hat{o})^{-1}-2}$. thus, we get, applying Lemma 4 that

$$
I_{\nu} \ll \frac{1}{d^{2}}\left[h\left(\frac{1}{2^{r-1}} K\right)^{(c+\delta)^{-1-2}}\right]^{-1 / 2} .
$$

We thus have for (12) the estimate:

$$
\ll \sum_{d \leqq A} \sum_{r} \sum_{\nu} \frac{1}{d^{2}} h^{-1 / 2} \frac{2^{(r-1) /(c+\delta) c}}{2^{r-1}} K^{1-1 /(2 c+2 \delta)}+\sum_{d \leqq A} \sum_{r}(E)
$$

(for largest $r$ we might get a shorter range of integration in (14), but the upper bound estimates still clearly hold in (16)). where $\sum_{\nu}$ is over

$$
\frac{1}{a(c+\delta)} h\left(\frac{1}{2^{r-1}} K\right)^{(c+\delta)-1-1} d^{2}-\frac{1}{2}<\nu<\frac{1}{a c} h\left(\frac{1}{2^{r}} K\right)^{c-1-1} d^{2}+\frac{1}{2} .
$$

From here, we have that the $\nu$ summation is bounded by

$$
\ll h d^{2} 2^{r}\left(\frac{1}{2^{r-1}}\right)^{1 /(c+\delta)} K^{c-1-1}+1
$$


and so we can further estimate (12) by

$$
\begin{aligned}
\ll & \sum_{d \leqq A} \sum_{r}\left(h d^{2} \frac{2^{r}}{2^{(r-1) /(c+\delta)}} K^{(1 / c)-1}+1\right)\left(\frac{h^{-1 / 2}}{d^{2}} \frac{2^{(r-1) /(2(c+\delta))}}{2^{r-1}} K^{1-1 /(2 c+2 \delta)}\right) \\
& +\sum_{d \leqq A} \sum_{r} E \ll A h^{1 / 2} K^{c^{-1-1 /(2 c+2 \delta)}}+h^{-1 / 2} K^{1-1 /(2 c+2 \delta)}+A \log K .
\end{aligned}
$$

(The last step is because $\Sigma_{r}$ in the first term was just a geometric sum and so it converges, while in the second part, the number of terms of the $\sum_{r}$ is $O(\log K)$.) The estimate $\left(16^{\prime}\right)$ together with (11) now gives us that

$$
T_{h}(K) \ll A h^{1 / 2} K^{(c+2 \delta) /(c+\delta) 2 c}+h^{-1 / 2} K^{1-1 /(2 c+2 \delta)}+\frac{K}{A}+K_{0} .
$$

Here, for $K$ sufficiently large the last error term absorbs into the first one if $A \geqq 1$ (which will anyway be the case). We now pick $A$ so as to balance the 1 st and 3 rd terms of (17), i.e. let $A=$

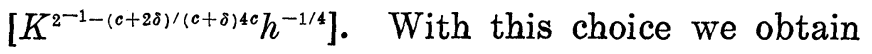

$$
T_{h}(K) \ll h^{1 / 4} K^{2-1+(c+2 \delta) /(c+\delta) 4 c}+h^{-1 / 2} K^{1-1 /(2 c+2 \delta)} .
$$

Finally we use Lemma 2 to write:

$$
\begin{aligned}
Q(K) D_{Q(K)}(g) & \ll \frac{K}{m+1}+\sum_{h=1}^{m} \frac{1}{h}\left\{h^{1 / 4} K^{2-1+(c+2 \delta) /(c+\delta) 4 c}+h^{-1 / 2} K^{1-1 /(2 c+2 \delta)}\right\} \\
& \ll \frac{K}{m+1}+m^{1 / 4} K^{2-1+(c+2 \delta) /(c+\delta) 4 c}+K^{1-1 /(2 c+2 \delta)} .
\end{aligned}
$$

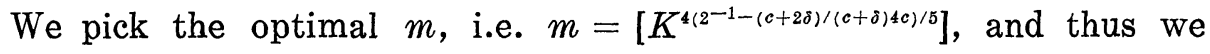
have

$$
Q(K) D_{Q(K)}(g) \ll K^{3 / 5+(c+2 \delta) /(c+\delta) 4 c}+K^{1-1 /(2 c+2 \tilde{o})}
$$

CoRollary 1. If $1<c<4 / 3$ then there exists $\delta_{0}>0$ depending on $c$ such that if $f(x) \in S(c, \delta)$ for $0<\delta<\delta_{c}$, and $g(x)$ is its inverse function then:

$$
Q(K) D_{Q(K)}(g) \ll K^{(c+\delta)^{-1-\varepsilon}} \ll(g(K))^{1-\varepsilon}
$$

for some $\varepsilon>0$, depending on $c$ and $\delta$.

Proof. All we need to show is that

$$
\frac{3}{5}+\frac{c+2 \delta}{5 c(c+\delta)}<\frac{1}{c+\delta} \text { and } 1-\frac{1}{2(c+\delta)}<\frac{1}{c+\delta}
$$

hold for some $\delta>0$. By continuity it is enough to check that $3 / 5+$ $1 / 5 c<1 / c$ and $1-1 / 2 c<1 / c$ hold. But the first of these holds if 
$c<4 / 3$, the second if $c<3 / 2$.

IV. We can now prove Theorem 1 . Let

$$
\begin{aligned}
T_{s}(x, y)= & \text { number of }\left\{s_{n}=[f(n)], y<s_{n} \leqq x, s_{n}=\right.\text { squarefree, } \\
& n=1,2,3, \cdots\}
\end{aligned}
$$

Clearly, $T_{s}(1, y)=Q_{s}(y)$. Lemma 1 can now be used together with expressions (6) and (21). $\xi$ in (6) will be taken $g^{\prime}(y+1)$ or $g^{\prime}(x-1)$ to give upper and lower bounds on $T(x, y)$, where $g(x)$ is as usual the inverse function of $f(x)$. We obtain:

$$
T_{s}(x, y)\left\{\begin{array}{l}
<g^{\prime}(x-1)(Q(y)-Q(x))+O\left(y^{(c+\delta)^{-1}-\varepsilon}\right) \\
>g^{\prime}(y+1)(Q(y)-Q(x))+O\left(y^{(c+\delta)^{-1}-\varepsilon}\right)
\end{array}\right.
$$

where $Q(x)=\#$ squarefree integers $\leqq x$. Or

$$
\begin{aligned}
& T_{s}(x, y) \\
& \left\{\begin{array}{l}
<g^{\prime}(x)(Q(y)-Q(x))+O\left(y^{(c+\delta)^{-1}-\delta}\right)+O\left(x^{c^{-1}-2}(Q(y)-Q(x))\right) \\
>g^{\prime}(y)(Q(y)-Q(x))+O\left(y^{(c+\delta)-1-s}\right)+O\left(y^{c^{-1}-2}(Q(y)-Q(x))\right) .
\end{array}\right.
\end{aligned}
$$

Thus, for $0<\alpha<1$, using the well-known fact that $Q(x)=6 \pi^{-2} x+$ $O\left(x^{1 / 2}\right)$,

$$
T_{s}(x,(1+\alpha) x)=\left\{\begin{array}{l}
<\frac{6}{\pi^{2}} x \cdot \alpha \cdot g^{\prime}(x) \\
>\frac{6}{\pi^{2}} x \cdot \alpha \cdot g^{\prime}((1+\alpha) x)
\end{array}+O\left(x^{(c+\delta)-1-\varepsilon}+x^{1 / 2}\right) .\right.
$$

On the other hand, clearly

$$
Q_{s}(x)=\sum_{k=1}^{L(x)} T_{s}\left(\frac{x}{(1+\alpha)^{k}}, \frac{x}{(1+\alpha)^{k-1}}\right)+O(1)
$$

holds for an appropriate function $L(x)$ which tends to $\infty$ for $x \rightarrow \infty$, if $\alpha=\alpha(x)>0$ is some given function of $x$ which tends to zero as $x \rightarrow \infty$ (the relation is $\left.(1+\alpha(x))^{L(x)} \cong x\right)$.

Using (25) in the expression (26) we obtain

$$
Q_{s}(x)\left\{\begin{array}{l}
<\frac{6}{\pi^{2}} \sum_{k=1}^{L(x)} \frac{x \cdot \alpha}{(1+\alpha)^{k}} g^{\prime}\left(\frac{x}{(1+\alpha)^{k}}\right)+O\left(L(x) \cdot x^{\gamma}\right) \\
>\frac{6}{\pi^{2}} \sum_{k=1}^{L(x)} \frac{x \cdot \alpha}{(1+\alpha)^{k}} g^{\prime}\left(\frac{x}{(1+\alpha)^{k-1}}\right)+O\left(L(x) \cdot x^{\gamma}\right)
\end{array}\right.
$$

where $\gamma=\max \{1 /(c+\delta)-\varepsilon, 1 / 2\}$ and so it is actually $1 /(c+\delta)-\varepsilon$. The main terms of the expressions on the right of (27) are exactly the upper and lower approximating sums of the Riemann integral 


$$
\int_{1}^{x} g^{\prime}(y) d y=g(x)-g(1) .
$$

To see how closely these sums approximate the integral, it suffices to find out how closely they are to each other, i.e. to estimate:

$$
\begin{aligned}
\Delta(x)= & \mid \sum_{k=1}^{L(x)} \frac{x \alpha}{(1+\alpha)^{k}} g^{\prime}\left(\frac{x}{(1+\alpha)^{k-1}}\right) \\
& -\sum_{k=1}^{L(x)} \frac{x \cdot \alpha}{(1+\alpha)^{k}} g^{\prime}\left(\frac{x}{(1+\alpha)^{k}}\right) \mid \\
= & \mid \sum_{k=0}^{L(x)-1} \frac{x \cdot \alpha}{(1+\alpha)^{k+1}} g^{\prime}\left(\frac{x}{(1+\alpha)^{k}}\right) \\
& -\sum_{k=1}^{L(x)} \frac{x \cdot \alpha}{(1+\alpha)^{k}} g^{\prime}\left(\frac{x}{(1+\alpha)^{k}}\right) \mid \\
\leqq & \left|\alpha \cdot \sum_{k=1}^{L(x)-1} \frac{x}{(1+\alpha)^{k}}\left(\frac{1}{1+\alpha}-1\right) g^{\prime}\left(\frac{x}{(1+\alpha)^{k}}\right)\right| \\
& +\left|x \cdot \alpha \cdot g^{\prime}(x)\right|+O(\alpha) \\
\ll & \left|\alpha \cdot \sum_{k=1}^{L(x)-1} \frac{x \cdot \alpha}{(1+\alpha)^{k+1}} g^{\prime}\left(\frac{x}{(1+\alpha)^{k}}\right)\right| \\
& +O\left|x \cdot \alpha \cdot g^{\prime}(x)\right|+O(\alpha) .
\end{aligned}
$$

The last sum is now the lower estimating sum of the integral, so one can write for $\alpha=\alpha(x)$

$$
\Delta(x) \ll \alpha(x)(g(x)-g(1))+\alpha(x) \cdot x \cdot g^{\prime}(x),
$$

so

$$
\Delta(x) \ll \alpha(x) g(x)+O(1) .
$$

Equation $(1+\alpha(x))^{L(x)} \cong x$ gives us that $\alpha(x)$ and $L(x)=[\log x / \alpha(x)]$ is a pair for which expression (26) holds; picking in particular $\alpha(x)=\left(\log x(g(x))^{-\varepsilon}\right)^{1 / 2}$, gives

$$
\Delta(x) \ll \sqrt{\log x}(g(x))^{1-\varepsilon / 2},
$$

and

$$
L(x) \cdot x^{\gamma} \ll L(x)(g(x))^{1-\varepsilon}=\sqrt{\log x}(g(x))^{1-\varepsilon / 2} .
$$

Calling $\varepsilon^{\prime}$ some value $0<\varepsilon^{\prime}<\varepsilon / 2$ yields

$$
Q_{s}(x)=\frac{6}{\pi^{2}} g(x)+O\left(\left(g(x)^{1-\varepsilon^{\prime}}\right) .\right.
$$

I take this opportunity to express my appreciation to Professor Harold N. Shapiro for his continued advice and valuable suggestions. 


\section{REFERENCES}

1. P. Erdös and P. Turán, On a problem in the theory of uniform distribution, Indagationes Math., 10 (1948), 370-378, 406-412.

2. I. I. Piateskii-Shapiro, On the distribution of prime numbers in sequences of form $\left[n^{c}\right]$, Math. Sbornik, 33 (1953).

3. E. C. Titchmarsh, The Theory of the Riemann Zeta Function, Oxford University Press, (1951).

Received June 30, 1975. This work was partially supported by the National Science Foundation, Grant NSF-GP-33019X.

Columbia University, New Yory City 


\title{
PACIFIC JOURNAL OF MATHEMATICS
}

\section{EDITORS}

RichaRd ARENS (Managing Editor)

University of California

Los Angeles, California 90024

\section{R. A. Beaumont}

University of Washington

Seattle, Washington 98105
J. DUGUNDJI

Department of Mathematics University of Southern California Los Angeles, California 90007

D. Gilbarg and J. Milgram

Stanford University

Stanford, California 94305

\section{ASSOCIATE EDITORS}
E. F. BECKENBACH
B. H. NeUmanN
F. WOLF
K. YoshIDA

\section{SUPPORTING INSTITUTIONS}

\author{
UNIVERSITY OF BRITISH COLUMBIA \\ CALIFORNIA INSTITUTE OF TECHNOLOGY \\ UNIVERSITY OF CALIFORNIA \\ MONTANA STATE UNIVERSITY \\ UNIVERSITY OF NEVADA \\ NEW MEXICO STATE UNIVERSITY \\ OREGON STATE UNIVERSITY \\ UNIVERSITY OF OREGON \\ OSAKA UNIVERSITY
}

\author{
UNIVERSITY OF SOUTHERN CALIFORNIA \\ STANFORD UNIVERSITY \\ UNIVERSITY OF TOKYO \\ UNIVERSITY OF UTAH \\ WASHINGTON STATE UNIVERSITY \\ UNIVERSITY OF WASHINGTON \\ AMERICAN MATHEMATICAL SOCIETY
}

The Supporting Institutions listed above contribute to the cost of publication of this Journal, but they are not owners or publishers and have no responsibility for its content or policies.

Mathematical papers intended for publication in the Pacific Journal of Mathematics should be in typed form or offset-reproduced, (not dittoed), double spaced with large margins. Underline Greek letters in red, German in green, and script in blue. The first paragraph or two must be capable of being used separately as a synopsis of the entire paper. Items of the bibliography should not be cited there unless absolutely necessary, in which case they must be identified by author and Journal, rather than by item number. Manuscripts, in triplicate, may be sent to any one of the editors. Please classify according to the scheme of Math. Reviews, Index to Vol. 39. All other communications should be addressed to the managing editor, or Elaine Barth, University of California, Los Angeles, California, 90024.

The Pacific Journal of Mathematics expects the author's institution to pay page charges, and reserves the right to delay publication for nonpayment of charges in case of financial emergency.

100 reprints are provided free for each article, only if page charges have been substantially paid. Additional copies may be obtained at cost in multiples of 50 .

The Pacific Journal of Mathematics is issued monthly as of January 1966. Regular subscription rate: $\$ 72.00$ a year (6 Vols., 12 issues). Special rate: $\$ 36.00$ a year to individual members of supporting institutions.

Subscriptions, orders for back numbers, and changes of address should be sent to Pacific Journal of Mathematics, 103 Highland Boulevard, Berkeley, California, 94708.

PUBLISHED BY PACIFIC JOURNAL OF MATHEMATICS, A NON-PROFIT CORPORATION

Printed at Kokusai Bunken Insatsusha (International Academic Printing Co., Ltd.), 8-8, 3-chome, Takadanobaba, Shinjuku-ku, Tokyo 160, Japan.

Copyright (C) 1975 by Pacific Journal of Mathematics Manufactured and first issued in Japan 


\section{Pacific Journal of Mathematics}

\section{Vol. 59, No. $2 \quad$ June, 1975}

Aharon Atzmon, A moment problem for positive measures on the unit disc ........

Peter W. Bates and Grant Bernard Gustafson, Green's function inequalities for

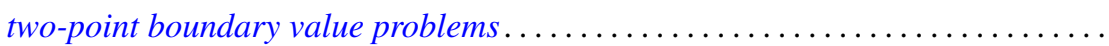

Howard Edwin Bell, Infinite subrings of infinite rings and near-rings ...........

Grahame Bennett, Victor Wayne Goodman and Charles Michael Newman, Norms of

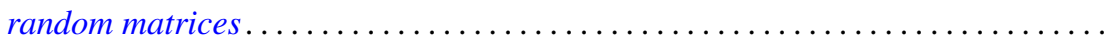

Beverly L. Brechner, Almost periodic homeomorphisms of $E^{2}$ are periodic.........

Beverly L. Brechner and R. Daniel Mauldin, Homeomorphisms of the plane ........

Jia-Arng Chao, Lusin area functions on local fields ......................

Frank Rimi DeMeyer, The Brauer group of polynomial rings ...............

M. V. Deshpande, Collectively compact sets and the ergodic theory of

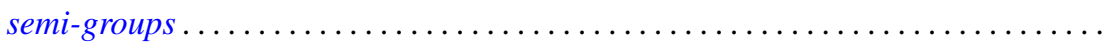

Raymond Frank Dickman and Jack Ray Porter, $\theta$-closed subsets of Hausdorff

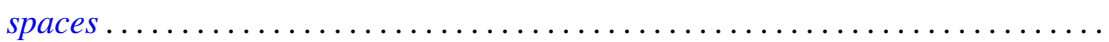

Charles P. Downey, Classification of singular integrals over a local field .......... 407

Daniel Reuven Farkas, Miscellany on Bieberbach group algebras . . . . . . . . . . . .

Peter A. Fowler, Infimum and domination principles in vector lattices . . . . . . . . . .

Barry J. Gardner, Some aspects of T-nilpotence. II: Lifting properties over

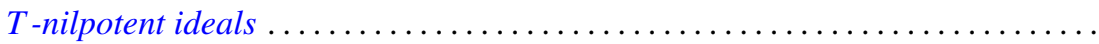

Gary Fred Gruenhage and Phillip Lee Zenor, Metrization of spaces with countable

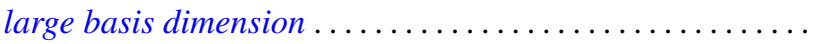

J. L. Hickman, Reducing series of ordinals...

Hugh M. Hilden, Generators for two groups related to the braid group ...

Tom (Roy Thomas Jr.) Jacob, Some matrix transformations on analytic sequence

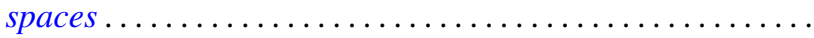

Elyahu Katz, Free products in the category of $k_{w}$-groups . .....

Tsang Hai Kuo, On conjugate Banach spaces with the Radon-Nikodým property...

Norman Eugene Liden, $K$-spaces, their antispaces and related mappings ...

Clinton M. Petty, Radon partitions in real linear spaces ........

Alan Saleski, A conditional entropy for the space of pseudo-Menger maps ....

Michael Singer, Elementary solutions of differential equations .

Eugene Spiegel and Allan Trojan, On semi-simple group algebras. I. . .

Charles Madison Stanton, Bounded analytic functions on a class of open Riemann

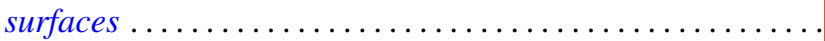

Sherman K. Stein, Transversals of Latin squares and their generalizations ....

Ivan Ernest Stux, Distribution of squarefree integers in non-linear sequences . . .

Lowell G. Sweet, On homogeneous algebras ................

Lowell G. Sweet, On doubly homogeneous algebras .......... .

Florian Vasilescu, The closed range modulus of operators ......

Arthur Anthony Yanushka, A characterization of the symplectic groups $\operatorname{PSp}(2 m, q)$

as rank 3 permutation groups... 